\title{
Assessment of cyclobenzaprine in the treatment of spasticity
}

\author{
PETER ASHBY, DAVID BURKE, SUDHAKAR RAO, \\ AND RICHARD F. JONES \\ From the Division of Neurology and the Department of Rehabilitation Medicine, \\ Prince Henry Hospital, Sydney, Australia
}

SUMMARY The efficacy of cyclobenzaprine $60 \mathrm{mg} /$ day in the treatment of spasticity was assessed in a double-blind crossover trial of two weeks' duration in 15 patients suffering from cerebral or spinal spasticity. Independent clinical and electromyographic methods were used. The effects of cyclobenzaprine did not differ significantly from those of placebo. The administration of a higher dosage, $150 \mathrm{mg} /$ day, to one patient revealed a dose-related response, but the degree of improvement was clinically small. Apart from a skin rash there were no significant untoward effects of therapy.

Pharmacological agents play a small but significant role in the management of the spastic patient. Effective spasmolytic agents are valuable in the early stages following spinal cord trauma where spasticity makes nursing management difficult, when independent use of a wheelchair is threatened by deforming postures, and, most importantly, in the maintenance of a degree of muscle tone which allows effective use of residual power. To fulfil these needs the development and testing of new drugs for the control of spasticity find their rationale.

After encouraging preclinical reports and apparent success in an initial pilot study in man (Merck, Sharp and Dohme Research Laboratories, personal communication), a double-blind trial was carried out to assess the efficacy of cyclobenzaprine hydrochloride (MK130) in the treatment of human spasticity. Cyclobenzaprine is a tricyclic amine related to amitriptyline. In animal experiments intravenous administration has been found to abolish the rigidity of both intercollicular decerebration ('gamma rigidity') and anaemic decerebration ('alpha rigidity'), and also to lessen the rigidity which follows spinal cord ischaemia. Unpublished clinical trials of cyclobenzaprine in the treatment of psychiatric disturbances (294 patients) and as a preanaesthetic medication (450 patients) have demonstrated that it is well tolerated by man, producing no untoward side-effects in oral dosage of up to $600 \mathrm{mg} /$ day and intravenous dosage of $15 \mathrm{mg}$ (Merck, Sharp and Dohme Research Laboratories, personal communication).

Assessment of the efficacy of medication for the relief of hypertonia must rely on clinical evaluation, despite potential observer inconsistency. The additional value of an objective assessment lies in its objectivity and in its potentially greater discriminative power. In this trial an objective assessment based on the electromyographic method used by Jones, Burke, Marosszeky, and Gillies (1970) has been carried out to complement and amplify the clinical findings. Although the results of this study are essentially negative, they are reported as a guide for possible future trials of this drug. In addition, a simple and reliable objective method of assessment of spastic hypertonia is further elaborated.

\section{METHODS}

Fifteen inpatients were studied. In five, aged 16 to 38 years, spasticity was due to cervical or thoracic spinal cord damage of at least nine months' duration. In two of these the lesion was clinically complete, and in all five the clinical condition was stable. In the remaining 10 patients, aged 8 to 69 years, brain damage was the aetiological factor, the causative lesion having taken place two to 18 months previously. Six of the 10 were hemiparetic, following cerebral throm- 
bosis (three patients), cerebral embolus (two patients), and subarachnoid haemorrhage (one patient). Four patients had suffered diffuse brain-stem disease in motor vehicle accidents, resulting in hemiparesis in two and quadriparesis in the remaining two patients.

A double-blind crossover study was carried out to compare the efficacy of cyclobenzaprine $60 \mathrm{mg} / \mathrm{day}$ with that of placebo. All sedatives, tranquillizers, and muscle relaxants in use before the trial were suspended for the duration of the study. Each treatment period was of two weeks' duration. Patients were randomly assigned to one treatment group, crossing over to the other after two weeks. Cyclobenzaprine was administered in $10 \mathrm{mg}$ tablets, $30 \mathrm{mg} /$ day in three divided doses for the first three days, and $60 \mathrm{mg} /$ day for the remainder of the treatment period. Placebo tablets were administered in an identical manner.

Clinical assessment was carried out daily by two of the authors (S.R. and R.F.J.), and trained physiotherapists made additional daily observations. The clinical assessment evaluated functional changes, muscle power, muscle tone, tendon hyperreflexia, and clonus. Voluntary power was graded using the Medical Research Council scale. Muscle tone was graded from no resistance (0), through slight (1), moderate (2), and marked resistance (3) to complete rigidity of the limb (4). Tendon reflexes were recorded as 0 (absent), + (reduced), ++ (normal), +++ (increased), and ++++ (markedly increased). The duration of muscle clonus was recorded in seconds, longer than $30 \mathrm{sec}$ being regarded as maximal. Disability scores were calculated by averaging the ratings of each examiner over the last five days of the treatment period. The significance of changes in these indices was assessed using Student's $t$ test.

The objective assessment was performed by two authors (P.A. and D.B.) on the last day of each treatment period, and was carried out completely independently of the clinical evaluation. There was no collaboration between authors until the completion of the study. The maximum force that could be developed by voluntary isometric contraction of the paretic quadriceps muscle was estimated with the knee joint at $90^{\circ}$ flexion, using a force transducer strapped to the limb above the ankle. The size of the quadriceps tendon jerk was measured under the same conditions, the mean of the largest 10 readings being documented. The significance of changes in voluntary power and of changes in the tendon jerk was assessed using Student's $t$ test.

A comparison of the relationship between the velocity of stretch and the resulting reflex response when on cyclobenzaprine with that when on placebo was used as an indication of the effect of cyclo- benzaprine on the stretch reflex. The quadriceps and hamstrings muscles were stretched passively by manual movements of the leg at the knee. Knee joint position was measured by a goniometer, the output of which was differentiated (time constant $5 \mathrm{msec}$ ) to produce a voltage proportional to angular velocity. The electromyogram (EMG) of quadriceps and hamstrings muscles was recorded by surface electrodes in standardized positions $15 \mathrm{~cm}$ apart over the relevant muscle. Such surface electrodes have been shown to provide the most reliable measurements of EMG in day to day testing (Buskirk and Komi, 1970). The of EMG records were monitored on an oscilloscope to detect artefact and to assess field spread of activity from the antagonistic muscle. The EMG potentials were integrated (time constant $0.2 \mathrm{sec}$ ), and recorded in both the raw and integrated forms, together with the records of joint position and angular velocity, on a four-channel Offner Dynograph (for quadriceps) or a four-channel Grass polygraph (for hamstrings).

The velocity of the stretching movement was plotted against the amplitude of the resulting integrated EMG and linear regression analysis was performed for those movements which produced EMG. of The EMG:velocity relationship could then be ex or pressed in terms of the intercept of the regressio 0 line on the velocity axis (the 'threshold velocity') an the slope of the regression line. Changes in the relationship produced by cyclobenzaprine were $\overrightarrow{0}$ taken to represent alterations in the sensitivity of the stretch reflex, and could be readily expressed as $\frac{Q}{8}$ 응 change in the threshold velocity or in the slope of the $\mathbb{\Phi}$ regression line. The EMG produced at $200^{\circ} / \mathrm{sec}$ w determined from the regression line and was used as a point of comparison for statistical purposes. The velocity $200^{\circ} / \mathrm{sec}$ was chosen because it has been noted empirically that if the threshold velocity exceeds $200^{\circ} / \mathrm{sec}$ it is usually difficult to detect increased muscle tone clinically.

Patients were specifically questioned each day to determine side-effects of the medication, with particular attention to anticholinergic effects. Other side-effects were looked for on physical examination, and in addition at the end of each trial period an electroencephalogram was performed and full blood count, serum electrolytes, serum creatinine, blood urea, and liver function tests were determined.

\section{RESULTS}

There was a general tendency for all patients with cerebral spasticity to improve slowly both $\frac{7}{0}$ physically and mentally throughout the month of the trial. This improvement may have resulted $\stackrel{\circ}{\circ}$ from more frequent passive movements and more $N$ 
active participation in the rehabilitation programme, but such a trend was not evident in the patients with spinal spasticity.

Of the 15 patients who started the trial 14 completed it satisfactorily, the fifteenth patient, quadriplegic from a cervical spinal cord lesion, withdrawing at the beginning of the second treatment period (cyclobenzaprine). It transpired that this patient had taken medication erratically throughout the trial and had been disappointed by the absence of improvement during the first treatment period (placebo). In a further patient the development of a rash resulted in premature termination of the treatment period with cyclobenzaprine after nine of the 14 days. However, in this patient full clinical and electromyographic

\section{CHANGE IN MUSCLE TONE}

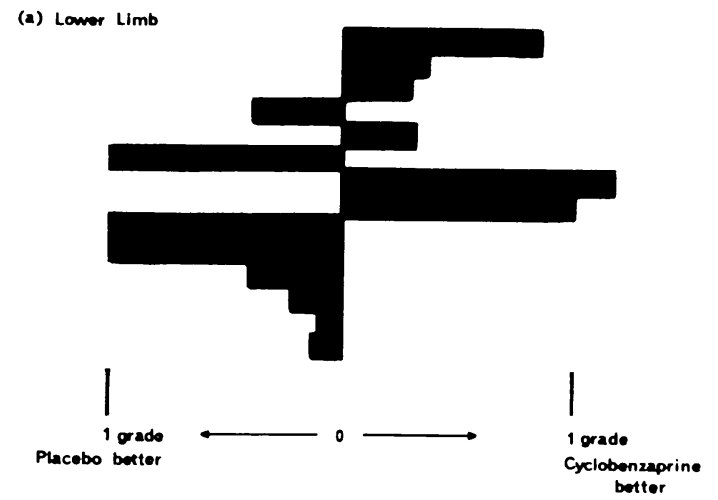

(b) Upper Limb

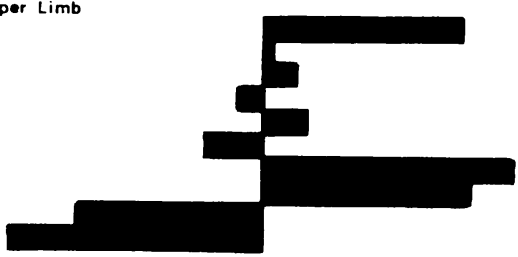

FIG. 1. Clinical assessment of changes in muscle tone. Changes in muscle tone were small, being rarely. more than one grade. For each patient the acerage difference between the assessments when on placebo and crclobenzaprine is shown. In (a) the changes in lower limb tone are shown, for the patients with cerebral spasticity first, and for the four patients with spinal spasticity in the lower part of the Figure. In (b) the changes in upper limb tone are shown for the 10 patients with cerebral spasticity, in the same order as in (a) observations were made before the suspension of therapy. Another patient, quadriplegic from trauma to the cervical spinal cord, completed the first treatment period (cyclobenzaprine) satisfactorily, although greatly troubled by severe spasticity. After four days on placebo in the second treatment period the frequency of muscle spasms had increased slightly, sufficient to cause suspension of this phase of the trial. Open treatment with cyclobenzaprine was started, the dosage being increased every second day from an initial $60 \mathrm{mg} /$ day to $150 \mathrm{mg} /$ day.

CLINICAL ASSESSMENT Three of the 14 patients appeared to be improved clinically when taking cyclobenzaprine, and another three seemed better when on placebo. There were minor changes in other patients, but these were small and of doubtful significance. The changes in muscle tone of the lower limb are shown diagrammatically in Fig. 1a. In the four spinal patients who completed the trial, the spinal cord lesion was either at or below the innervation of the upper limb muscles. Alterations in tone of upper limb muscles were therefore calculated only in the 10 cases of cerebral spasticity. These changes, seen in Fig. 1b, correlate well with those of the lower limb in the same patients.

There was no statistically significant difference between the effects of cyclobenzaprine and placebo on upper limb tone in the 10 patients $(t=0 \cdot 75)$, or on lower limb tone in the total of 14 patients $(t=0 \cdot 103)$. If only the 10 patients with cerebral spasticity were considered, the effects of cyclobenzaprine on lower limb tone still did not differ significantly from those of placebo $(t=$ $0 \cdot 223$ ).

When compared with placebo, cyclobenzaprine appeared to have no effect on voluntary power and, in the dosage of $60 \mathrm{mg} /$ day, did not alter the frequency of muscle spasms in four of the five patients who were prone to spasms. In one patient. cyclobenzaprine $150 \mathrm{mg}$ day did reduce the frequency of spasms. The effect on deep tendon reflexes was variable, scores being lower in five patients. higher in three patients, and unaltered in the remaining six patients. Of eight patients with clonus, the duration of clonus when on cyclobenzaprine was less in two patients, more in one patient and unchanged in five patients. 


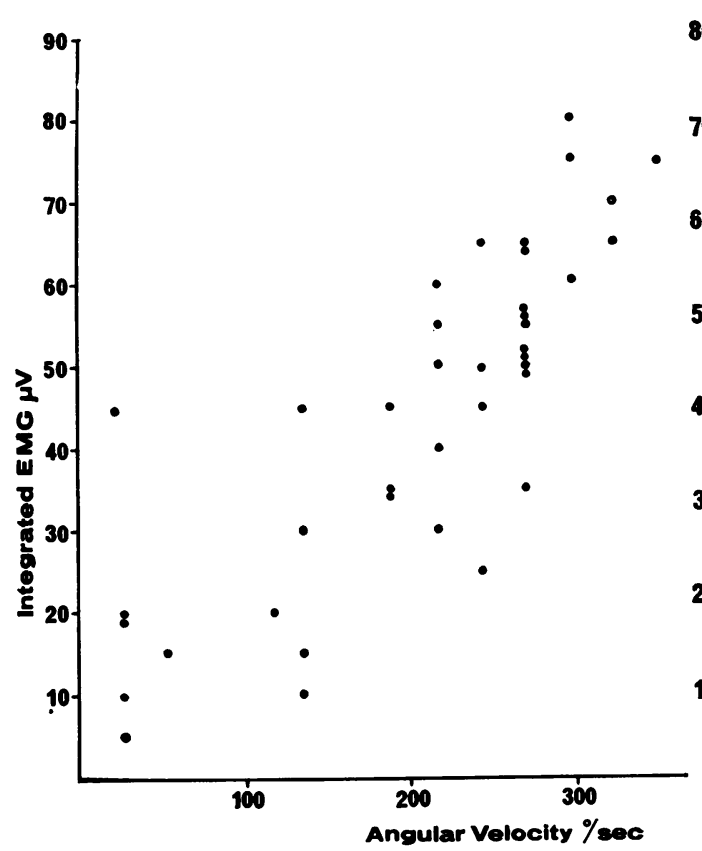

(a)

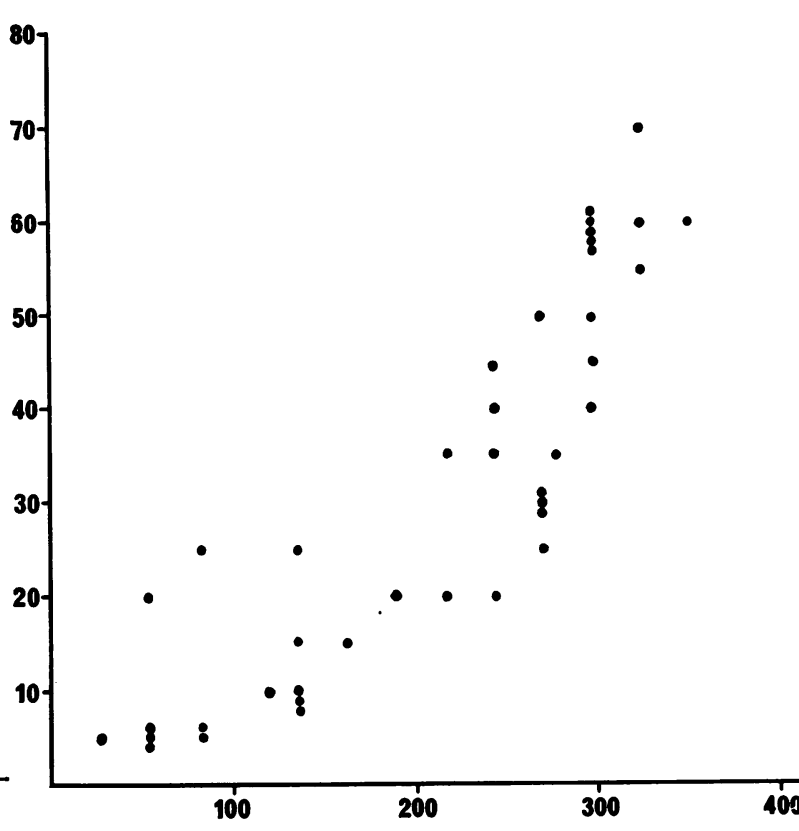

(b)

FIG. 2. The effect of cyclobenzaprine on the hamstrings stretch reflex of a patient with cerebral spasticity The EMG:velocity relationship obtained when on placebo is shown in (a). There was a slight reduction of reflexp activity when on cyclobenzaprine (b), shown by the displacement of the EMG:velocity relationship to the right $\overrightarrow{0}$ These graphs, and those of Fig. 3, illustrate the scatter of readings obtained in an average case.

The dosage of cyclobenzaprine was increased to $150 \mathrm{mg} /$ day in one patient with spinal spasticity after the suspension of the placebo treatment period due to uncontrollable spasms. This higher dosage resulted in a slight reduction in the intensity of spasticity, manifested by an average reduction in muscle tone of 0.5 grade, subjective decrease in the frequency and intensity of spasms, and diminution in tendon reflexes by 0.5 to 1 grade. No functional improvement resulted.

OBJECTIVE ASSESSMENT The EMG: velocity retionship of both the hamstrings and quadriceps muscles was approximately linear on both testings in most patients, as seen in Figs $2 \mathrm{a}, \mathrm{b}$ and $3 \mathrm{a}$. Occasionally the relationship was not best described by linear regression analysis (Fig. 3b), and in such instances the line of best fit was drawn visually.

Electromyographic results confirmed those of the clinical assessment. Using the EMG produced at $200^{\circ} / \mathrm{sec}$ as a point of statistical com- parison, and assuming that changes of $20 \%$ o less are insignificant, five patients improved on $N$ cyclobenzaprine, three appeared better on placebo, and in six patients there was no significant change in reflex status. Considering all 14 patients, the average change produced by cyclobenzaprine was an improvement of $6 \%$, but this figure rose to $15 \%$ if only patients with cerebral spasticity were considered. Neither figure achieved statistical significance $(t=0.43$ and 0.79 respectively).

Cyclobenzaprine in a dose of $150 \mathrm{mg}$ /day produced a significant change in electromyographic criteria of spasticity in the one patient to whom this dose was administered. The slight clinical response to the standard dosage of $60 \mathrm{mg} / \mathrm{day}$ was reflected in the electromyographic examinations, improvement measuring $18 \%$. On administration of cyclobenzaprine $150 \mathrm{mg} /$ day a $63 \%$ reduction in reflex EMG occurred when compared with placebo. This improvement resulted predominantly from effects on the quadriceps 


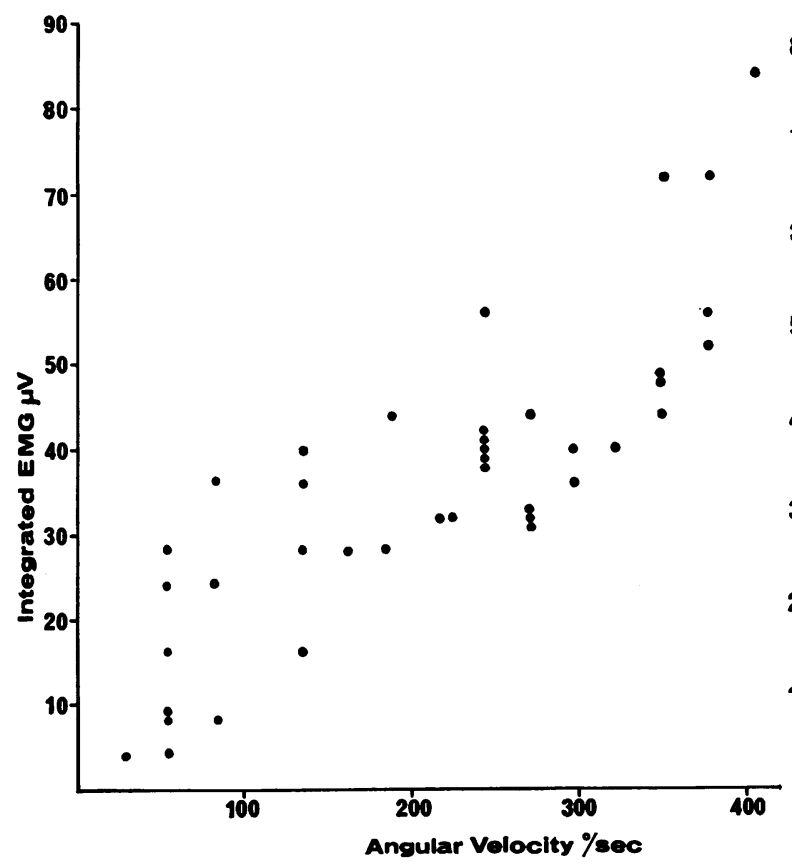

(a)

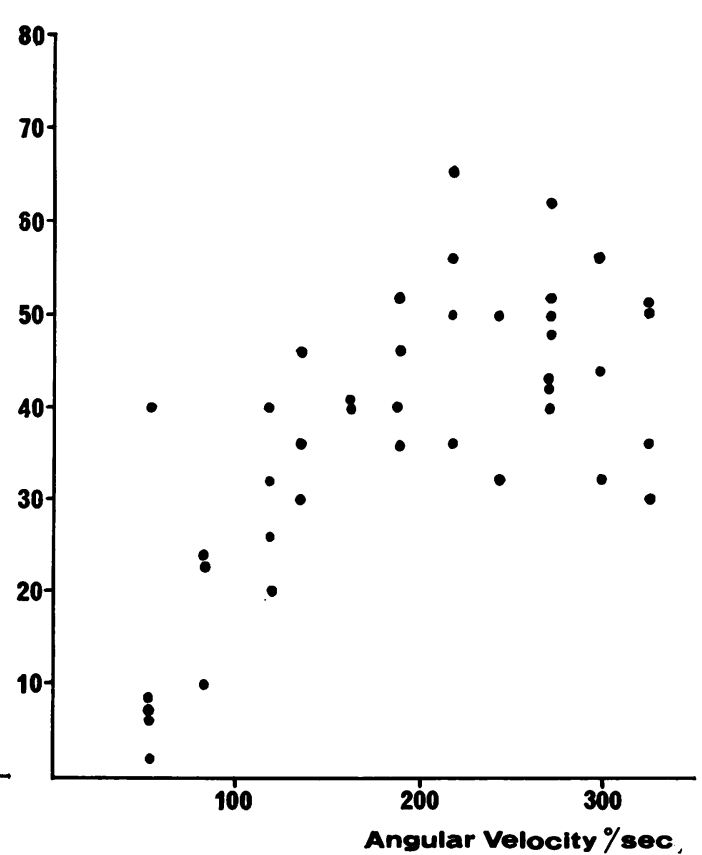

(b)

FIG. 3. The effect of cyclobenzaprine on the quadriceps stretch reflex in the same patient as in Fig. 2. The graphs are practically superimposable, there being little overall difference at any particular velocity of stretch between the relationship obtained when on placebo (a) and that when on cyclobenzaprine (b). The relationship in (b) is obviously non-linear, and is therefore unsuitable for linear regression analysis.
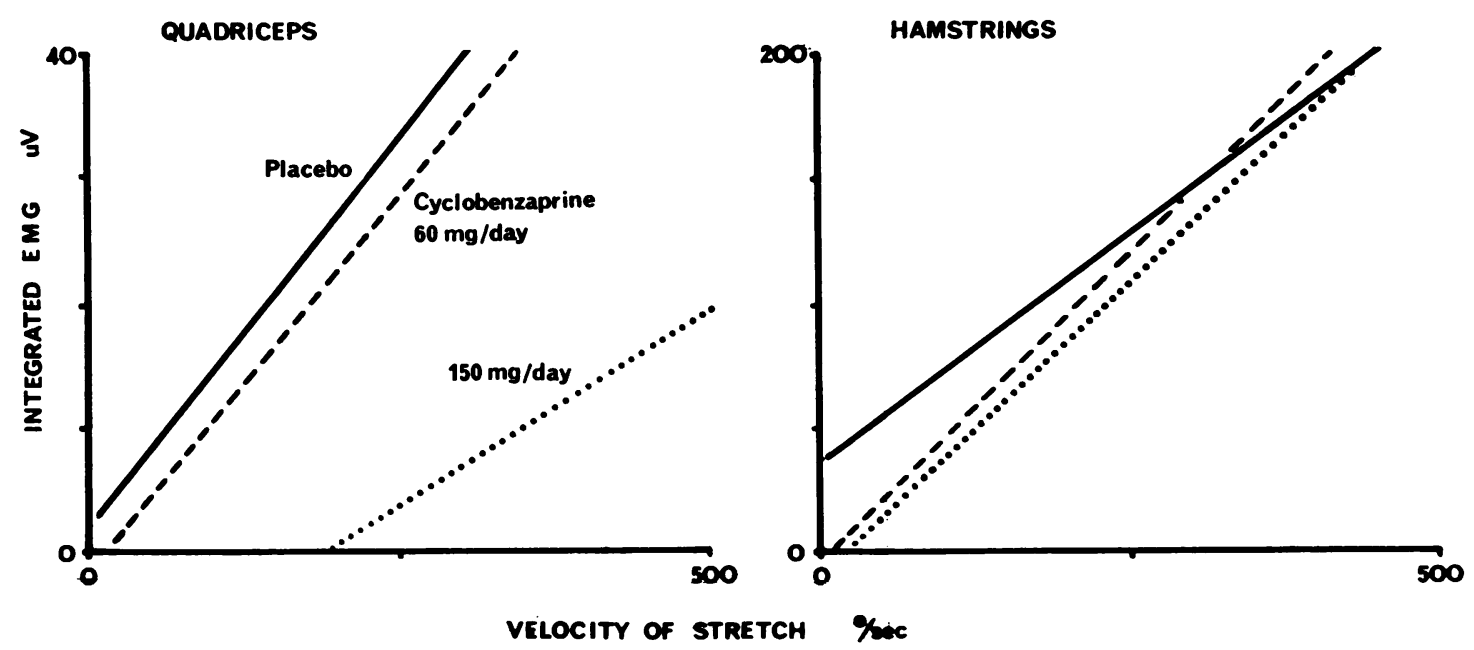

FIG. 4. The effect of higher doses of cyclobenzaprine. At the dosage of $60 \mathrm{mg} /$ day there is very little difference from placebo, although the EMG:velocity relationships suggest that there is slightly less reflex activity. At the dosage of $150 \mathrm{mg} /$ day there is little change in the hamstrings stretch reflex, but reflex activity is significantly decreased in the quadriceps muscle, seen as an increase in the threshold velocity and a decrease in the slope of the EMG:velocity relationship. 
stretch reflex (Fig. 4), in which the threshold velocity increased from $16^{\circ} / \mathrm{sec}$ when on cyclobenzaprine $60 \mathrm{mg} /$ day to $191^{\circ} / \mathrm{sec}$ when on 150 $\mathrm{mg} /$ day. There was a similar but minor effect on the hamstrings stretch reflex, threshold velocity increasing from $11 \% \mathrm{sec}$ when on $60 \mathrm{mg} /$ day to $23^{\circ} / \mathrm{sec}$ when on $150 \mathrm{mg} /$ day (Fig. 4).

The effect of cyclobenzaprine on the force produced by maximal voluntary contraction of the paretic quadriceps muscle was assessed in all 10 patients with cerebral spasticity and the one spinal patient who retained some voluntary power in the affected quadriceps muscle. When taking cyclobenzaprine the force recorded was reduced in six patients, increased in four patients and unaltered in one patient. The average effect was a reduction of $0.6 \mathrm{~kg}$, an effect which was not statistically significant $(t=0 \cdot 33)$. Cyclobenzaprine reduced the amplitude of the knee jerk by an average of $0.33 \mathrm{~kg}$. Again this effect did not achieve statistical significance $(t=0 \cdot 21)$.

SIDE-EFFECTS The administration of cyclobenzaprine did not alter the biochemical screening tests, and on clinical examination no side-effects were apparent, apart from a pruritic truncal maculopapular rash which necessitated cessation of the cyclobenzaprine treatment period in one spinal patient. Another patient developed a truncal rash during placebo treatment.

A number of minor subjective symptoms were elicited on interrogation, but these were transient and most occurred in both treatment periods. One patient developed a dry mouth when on cyclobenzaprine but this cleared spontaneously after one day. A second patient complained of dizziness when receiving cyclobenzaprine and this patient also experienced nausea and vomiting when on placebo. One patient suffered attacks of nausea and vomiting in both treatment periods, but these persisted for only two days on each occasion.

The electroencephalograms of most patients with cerebral spasticity and one with spinal spasticity were abnormal, but the records taken in the two treatment periods did not differ significantly.

\section{DISCUSSION}

While it is probable that cyclobenzaprine has some slight activity in reducing spasticity, the results of this trial suggest that it is likely to be of $z$

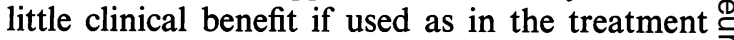
regime adopted for this trial. The failure of cyclobenzaprine to be of significant benefit to $\frac{c}{0}$ spastic patients may be in part due to the limita- 0 tion of dosage. In the one patient in whom dosage $\stackrel{\infty}{\circ}$ was increased as far as $150 \mathrm{mg} /$ day a doserelated response was found electromyographically, but even at this dosage level clinical improvement was not impressive.

Cyclobenzaprine is related to amitriptyline. As such it is possible that its full therapeutic effect may take a comparatively long time to become manifest, perhaps longer than was allotted in this trial. However there was no change over the last $\%$ few days of the cyclobenzaprine treatment period $\overrightarrow{0}$ to suggest that significant improvement would have occurred had treatment been continued. It $\stackrel{\omega}{\sigma}$ is possible that cyclobenzaprine may prove to be a useful adjuvant to other drug therapy, but this possibility was not investigated in this trial, other of relevant drugs being suspended for its durationo $r$ Thus, at this stage, it seems that unless the resula of high dosage, long-term therapy are more img pressive, cyclobenzaprine offers little that is ne and has no place in the treatment of spasticity 0

Cyclobenzaprine appears to be well tolerateg by man, even in high dosage. If further studies this drug are to be undertaken, the negative re sults of this trial would suggest that it be adminit stered in higher dosage for longer treatment periods possibly in the presence of other muscle relaxants.

The most gratifying result of this trial was the high degree of correlation between the clinical findings and the electromyographic assessment. The electromyographic studies were based on two essentially simple principles: that the spastic stretch reflex is a dynamic stretch reflex in which the reflex response is approximately linearly related to the velocity of stretch (Burke, Gillies, and Lance, 1970, 1971), and that changes in muscle tone are manifested by changes in the relationship between the velocity of stretch and the reflex response. Both in this study and in earlier ones from this laboratory (Jones et al., 1970; 은 Burke, Andrews, and Knowles, 1971), there $\frac{D}{0}$ appears to be ample evidence to substantiate both principles. The equipment required for the $\mathcal{N}$ electromyographic studies is neither complex nor $N$ 
beyond the scope of most clinical neurophysiological laboratories. It is suggested that the methods used provide a simple technique for assessing objectively changes in clinical status, be they due to the passage of time or to therapeutic intervention.

The authors are grateful to Associate Professor James W. Lance for guidance, and for help with the preparation of this report, and to Miss A. M. Mackie, Chief Pharmacist of the Prince Henry Hospital, for kindly arranging the random allocation of cyclobenzaprine and placebo for this trial. We would also like to thank the nursing staff and physiotherapists of the Department of Rehabilitation Medicine, and Dr. V. J. Hercus, Medical Director, Merck, Sharp and Dohme (Aust.) Pty. Ltd. for supplies of cyclobenzaprine and placebo tablets and for invaluable assistance throughout the trial.

\section{REFERENCES}

Burke, D., Andrews, C. J., and Knowles, L. (1971). The action of a GABA derivative in human spasticity. Journal of Neurological Sciences, 14, 199-208.

Burke, D., Gillies, J. D., and Lance, J. W. (1970). The quadriceps stretch reflex in human spasticity. Journal of Neurology, Neurosurgery, and Psychiatry, 33, 216-223.

Burke, D., Gillies, J. D., and Lance, J. W. (1971). Hamstrings stretch reflex in human spasticity. Journal of Neurology, Neurosurgery, and Psychiatry, 34, 231-235.

Buskirk, E. R., and Komi, P. V. (1970). Reproducibility of electromyographic measurements with inserted wire electrodes and surface electrodes. Acta Physiologica Scandinavica, 79, 29A.

Jones, R. F., Burke, D., Marosszeky, J. E., and Gillies, J. D. (1970). A new agent for the control of spasticity. Journal of Neurology, Neurosurgery, and Psychiatry, 33, 464-468. 